\title{
NEUTRON GENERATORS DEVELOPED AT LBNL FOR HOMELAND SECURITY AND IMAGING APPLICATIONS*
}

\author{
J. Reijonen", E. O. Lawrence Berkeley National Laboratory, Berkeley CA 94720, USA
}

\begin{abstract}
The Plasma and Ion Source Technology Group at Lawrence Berkeley National Laboratory has developed various types of advanced D-D (neutron energy $2.5 \mathrm{MeV}$ ), $\mathrm{D}-\mathrm{T}(14 \mathrm{MeV})$ and T-T (0-9 MeV) neutron generators for wide range of applications. These applications include medical (Boron Neutron Capture Therapy), homeland security (Prompt Gamma Activation Analysis, Fast Neutron Activation Analysis and Pulsed Fast Neutron Transmission Spectroscopy) and planetary exploration with a sub-surface material characterization on Mars. These neutron generators utilize RF induction discharge to ionize the deuterium/tritium gas. This discharge method provides high plasma density for high output current, high atomic species from molecular gases, long life operation and versatility for various discharge chamber geometries. Four main neutron generator developments are discussed here: high neutron output co-axial neutron generator for BNCT applications, point neutron generator for security applications, compact and sub-compact axial neutron generator for elemental analysis applications. Current status of the neutron generator development with experimental data will be presented.
\end{abstract}

\section{INTRODUCTION}

The Plasma and Ion Source Technology Group at the E. O. Lawrence Berkeley National Laboratory has been developing high power D-D neutron generators for various applications for more than ten years. The main difference between the LBNL neutron generators in comparison to the commercially available neutron generators is the way the plasma is generated in the generator. The discharge method utilized in the LBNL neutron generators is based on RF-induction discharge, a method primarily used in fusion energy research and in semiconductor industry. Although the RF-induction based neutron generators have been used before, the modern time development has created ion sources with unparallel reliability and stability of operation. Development of these RF-induction based ion source was started in the P\&IST group more than two decades ago. This RFtechnology in combination with unique RF-impedance matching circuitry and source designs capable of high power operation has enabled high yield D-D, D-T and T$\mathrm{T}$ neutron generators. These ion sources are currently used in three types of neutron generators; axial, single-beam neutron generator; co-axial, multi-beam, high current neutron generator; and point neutron generator with high

\footnotetext{
"This Work was supported by Tensor Technology Inc., by Adelphi Technology Inc. and by U.S. Department of Energy under contract No. DE-AC02-05CH11231.

"Email: jreijonen@1bl.gov
}

instantaneous neutron yields. In this presentation, the progress on the single-beam axial neutron generator and the point neutron generator for T-T wide energy neutron generation will be presented.

\section{AXIAL, SINGLE-BEAM D-D NEUTRON GENERATOR}

The high voltage shielded RF-induction ion source driven, axial D-D neutron generator[1] is a development of the previously developed sectioned accelerator[2] single beam D-D neutron generator. The fully high voltage shielded construction of the latest axial neutron generator is the main difference between these two neutron generators. It utilizes an external antenna RFinduction ion source for highly efficient plasma generation. It enables placement of the neutron generator, even in places where the moderator material next to the neutron generator is conducting. On the other hand, the irradiated samples can be placed next to the generator, to maximum neutron flux for activation experiments of various conducting or non-conducting samples. The use of co-axial, shielded high voltage cable minimizes the electromagnetic noise due to sparking and does not allow RF-energy to be induced at the cable. This translates into stable operation both, the neutron generator and the instruments close by the generator.

In the sectioned insulator design, the bare insulated high voltage cable was attached to an external voltage divider stack or the voltage was divided by using the target water-cooling lines as a series resistor stack between the accelerator stages. In the high voltage shielded design a co-axial high voltage cable is semipermanently attached to the target assembly. In order to keep the length of the cable short, the HV insulator is filled with dielectric transformer oil. The target cooling water is hosed in the same oil-filled insulator cylinder space. These target cooling-water hoses are coiled around the HV-cable guide in order to increase the total length of the lines to minimize the leakage current along the cooling-water. To further minimize the leakage Low Conductance Water (LCW) is used to cool the target. The main components of the high voltage shielded neutron generator is highlighted in Figure 1.

Two versions of the universal neutron generator were designed: one generator for the Department of Nuclear Engineering of University of California for educational purposes and another one for material analysis group at Korean Atomic Energy Research Institute (KAERI) for Neutron Activation Analysis and Prompt Gamma Activation Analysis applications. The main difference between these two neutron generators is the high voltage insulator design. While the UCB generator has a standard 
$50 \mathrm{~mm}$ in length, alumina $\left(\mathrm{Al}_{2} \mathrm{O}_{3}\right)$ vacuum insulator, the KAERI generator has a custom designed $150 \mathrm{~mm}$ in length alumina insulator, coated with semi conducting $\mathrm{TiCrO}_{3}$ coating. The proprietary coating process, marketed by Elcon Inc. of Santa Clara CA, provides excellent high voltage stability of an insulator operating in a vacuum volume with free charged particles, by draining charges away from the surface to ground, before they can are accumulated on the insulator, leading to high voltage break-downs. The KAERI neutron generator was tested with extracted hydrogen beam up to $120 \mathrm{kV}$ and $5 \mathrm{~mA}$ of beam current. The extracted hydrogen beam current as a function of accelerator voltage is plotted in Figure 2.

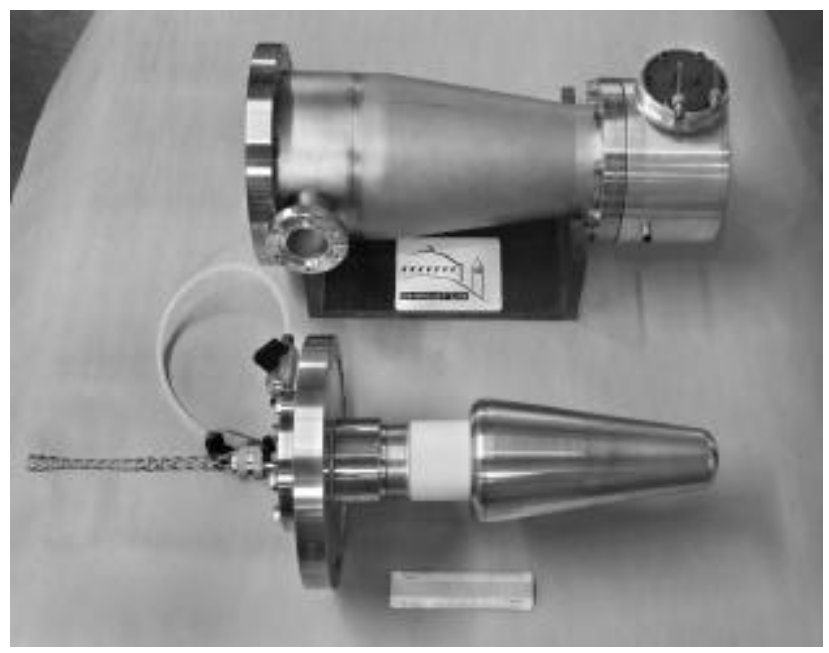

Figure 1: High voltage shielded, axial neutron generator shown with it's target assembly separated from the main vacuum chamber. The short, $50 \mathrm{~mm}$ high voltage insulator is shown in the picture.

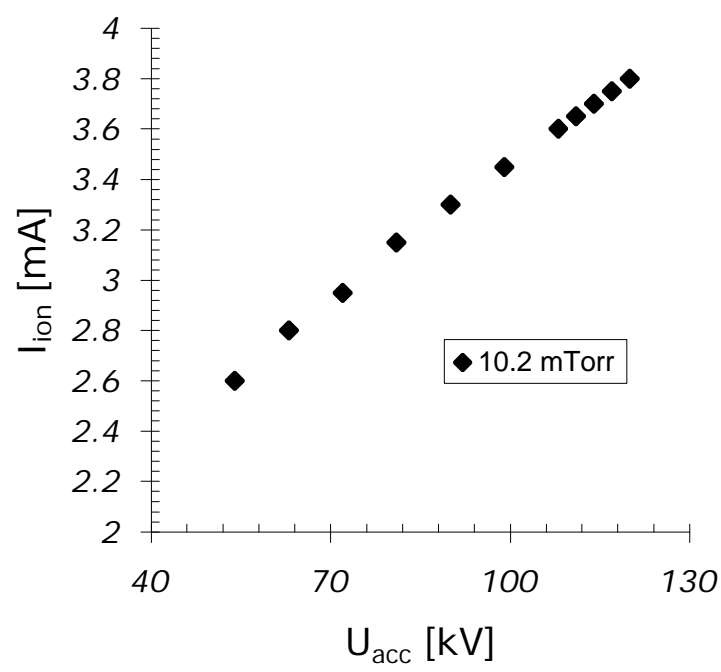

Figure 2: Extracted hydrogen beam current from the KAERI axial neutron generator. The ion source has $4 \mathrm{~mm}$ in diameter extraction aperture. Predicted D-D neutron yield is $>5 \times 10^{8} \mathrm{n} / \mathrm{s}$.
The UCB neutron generator has been extensively tested. The purpose is to build a relatively low RF-power neutron generator, which could produce D-D neutron yield of $>10^{7} \mathrm{n} / \mathrm{s}$. The UCB neutron generator is limited in performance in comparison to the KAERI neutron generator, even though the appearance of these two neutron generators is similar. The high voltage insulator is shorter in the UCB neutron generator. The maximum operation voltage is thus limited to $90 \mathrm{kV}$. Limited RFdischarge power means, the generator is operated at $<1$ $\mathrm{kW}$ of RF-discharge power. The neutron yield measurements shown here are made with $600 \mathrm{~W}$ of RFdischarge. The extracted beam current density is $\sim 12$ $\mathrm{mA} / \mathrm{cm}^{2}$ with atomic species fraction of the deuterium beam $>90 \%$, see Figure 3 .

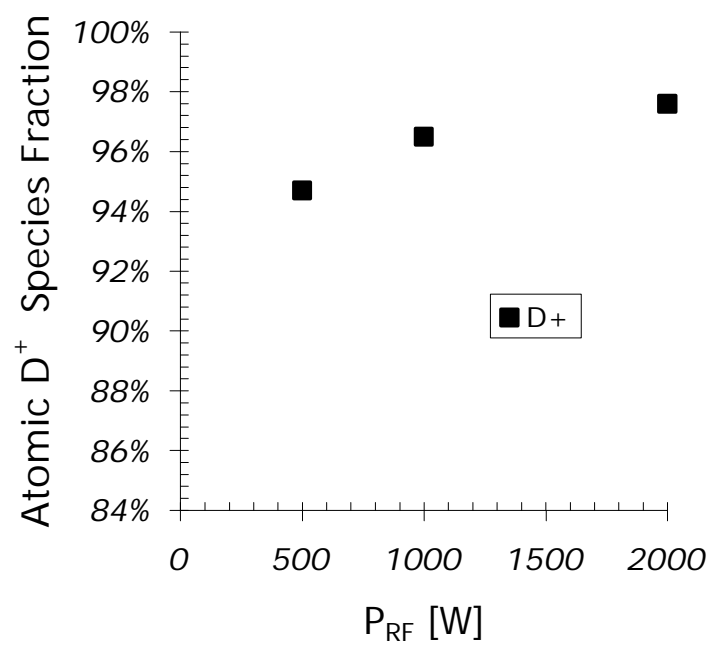

Figure 3: The fraction of atomic extracted deuterium ion beam from the total beam current as a function of RFdischarge power. Even low discharge power provides highly atomic species fraction for high efficiency neutron production

The ion optics has been simulated with IGUN 7.030 ion extraction/transport and plasma simulation code[3], see Figure 4. The aim for the simulations has been to achieve large enough beam spot size for low power density at the beam spot on the flat target surface so that the surface temperature on the target is less than $200{ }^{\circ} \mathrm{C}$. For higher surface temperatures desorption of the deuterium from the titanium matrix, will decrease the neutron output. The surface temperature on the target is fairly dfficult to measure directly, especially when the target is being actively water cooled. For the surface temperature analysis, engineering modeling program ANSYS was used. The IGUN simulations results were used for the beam spot diameter and for beam power density estimations. The results are shown in Figure 5, where the surface temperature of the 500 micron thick, explosive bonded titanium on copper bi-metal target from Atlas Inc. is analyzed. The beam has beam power density of 840 


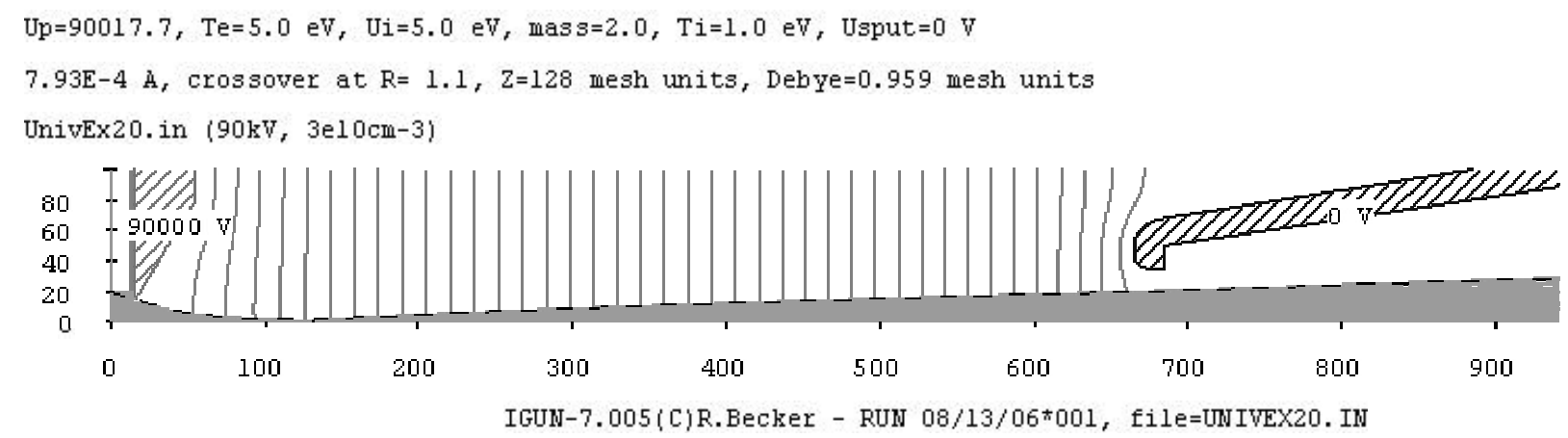

Figure 4. IGUN simulation of the beam extraction and optics in the UCB neutron generator. In this simulation, beam generated with $600 \mathrm{~W}$ of RF-discharge power is being simulated.

$\mathrm{W} / \mathrm{cm}^{2}$ and beam spot diameter size of $3.2 \mathrm{~mm}$. The surface temperature is only $151^{\circ} \mathrm{C}$.

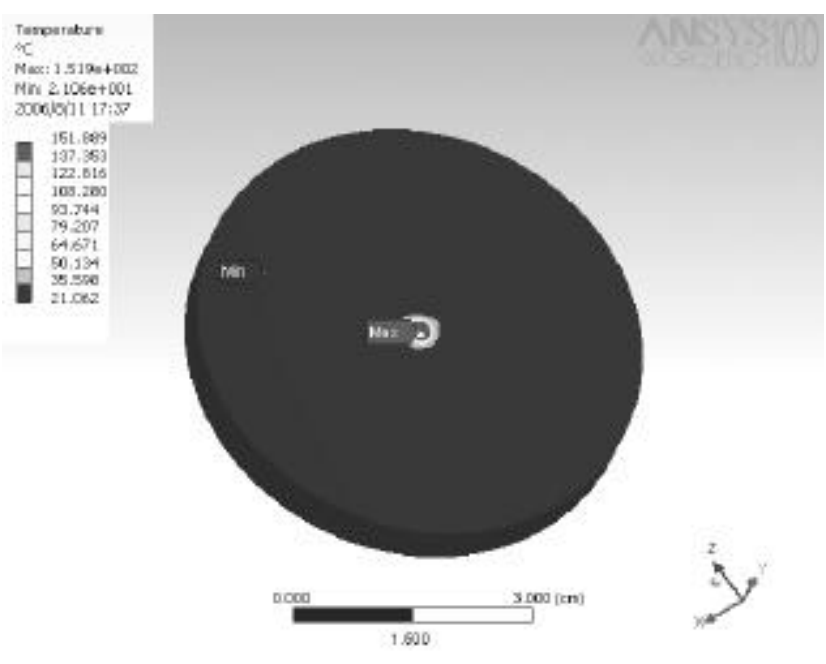

Figure 6: Surface temperature analysis of the bi-metal target surface. For $840 \mathrm{~W} / \mathrm{cm}^{2}$ power density the surface temperature is $\sim 150{ }^{\circ} \mathrm{C}$. Low surface temperature provides good titanium matrix loading.

The neutron yield was measured using Indium-foil activation analysis. For $90 \mathrm{kV}$ beam energy and $\sim 800$ micro-amperes of deuterium current the neutron yield is $3 \times 10^{7} \mathrm{n} / \mathrm{s}$. In comparison, the theoretical value can be obtained by using the well-known D-D cross-sections and calculating the ranges using SRIM code[4]. The measured yield compares reasonably well with the theoretical value of $7 \times 10^{7} \mathrm{n} / \mathrm{s}$, for the same current, in case were the titanium matrix is loaded with one deuterium per titanium. The difference in yield is resulting from beam losses due to neutralization of the ion beam in the accelerator gap, and/or the Ti/D ratios of $<1$ in the target Titanium matrix. These losses can be further minimized by operating the neutron generator at lower gas pressure and/or having smaller beam power density at the target surface.

\section{NEUTRON IMAGING NEUTRON GENERATOR}

The Plasma and Ion Source Technology Group has been collaborating with Adelphi Technology Inc. to develop a neutron imaging neutron generator. Neutrons can be used to image fine cracks and other metal pipe deformations in cooling pipes of power-plants and other critical applications[5]. For neutron imaging, a bright neutron source is desirable. This means, that for a neutron generator the beam spot size on the target has to be small and the beam power has to be high. In case of flat target, this leads to high surface temperatures and low neutron yield. One of the unique features of the D-D imaging neutron generator is a conical-shape target. The coneshape increases the cooling surface area of the target, allowing a small beam spot size on the axis of the neutron generator. The design specification for the imaging neutron generator (ING) is to have a D-D neutron yield $\sim 10^{9} \mathrm{n} / \mathrm{s}$, with a maximum of $3 \mathrm{~mm}$ in diameter beam spot size using $10 \mathrm{~mA}$ of beam current and $120 \mathrm{kV}$ of acceleration voltage. For flat target this would mean a beam power density of $\sim 14 \mathrm{~kW} / \mathrm{cm}^{2}$. If a cone-shape target is used instead with $50 \mathrm{~mm}$ of beam-swept depth, the power density at the surface is only $\sim 500 \mathrm{~W} / \mathrm{cm}^{2}$. Beam power of $1.2 \mathrm{~kW}$ can be comfortable cooled with water using a high voltage feed-through structure developed for the KAERI/UCB neutron generator. The ING is shown in Figure 7. The target is fabricated by a proprietary process to obtain thin titanium layer inside the cone, supported by relatively thick wall of copper. The target is then brazed into a holder structure, where the cooling water is flown.

Another innovation in the ING is the RF-induction ion source. Instead of operating a solenoid antenna on watercooled alumina cylinder, the RF-antennas are in a spiral shape on a flat RF-window. This arrangement has shown to provide high plasma densities at low operation pressure[6]. Both properties needed for sealed, or semisealed operation. The ING neutron source has a further development of the single antenna source by using two antennas, located perpendicular to the ion beam axis. This 
way the heat generated from the secondary electrons impinging the back plate can be easily handled. These quartz RF-windows are actively water-cooled by flowing water on the edge of the windows. Ion source with dual spiral antennas are now being tested at the Plasma and Ion Source Technology group. Rest of the ING will be fabricated and tested during the last quarter of 2006.

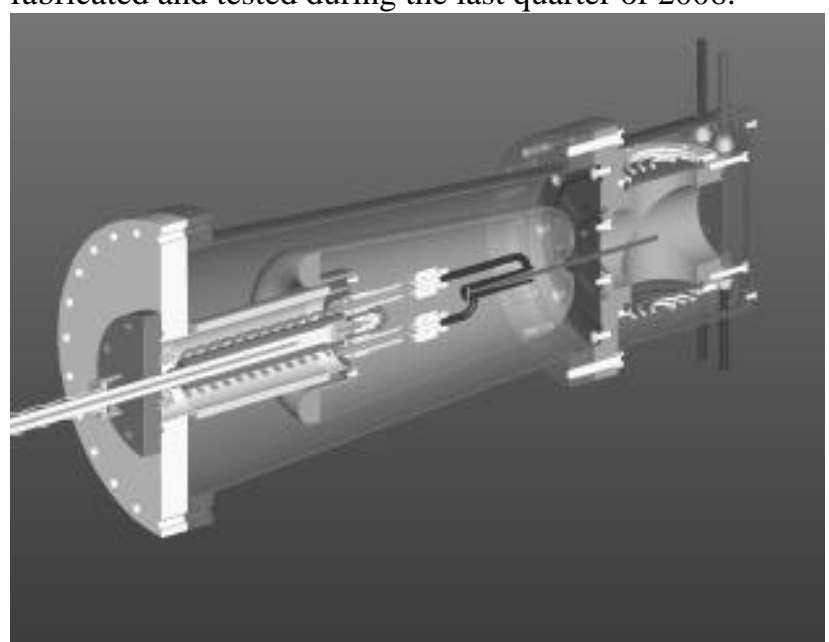

Figure 7. Imaging neutron generator with high power density cone target and external, spiral antenna ion source. The neutron generator is $25 \mathrm{~m}$ in diameter and 60 $\mathrm{cm}$ in length.

\section{POINT NEUTRON GENERATOR}

The progress of the $\mathrm{T}-\mathrm{T}$ point neutron generator has been reported earlier[1]. The purpose is the fabricate a highly specialized neutron generator the Tensor Technology Inc's Pulsed Fast Neutron Transmission Spectroscopy (PFNTS)-based, explosives screening system for airline cargo. The detection method is described in detail in reference[7]. The detection scheme requires a point neutron source for imaging, short neutron pulses for Time-of-Flight neutron energy measurements and wide energy spectrum for elemental identification using neutron resonance absorption technique. These requirements can be fulfilled with a neutron generator with ns-range pulsing capability, operating in fully $\mathrm{T}-\mathrm{T}$ mode to generate wide energy spectrum neutrons. Due to relatively low reaction cross-section of the $\mathrm{T}-\mathrm{T}$ fusion reaction, the neutron generator has to have high instantaneous beam current capability. The specifications of the Tensor Point White Neutron Generator (PWNG) is maximum of $1 \mathrm{~A}$ of beam current at $120 \mathrm{kV}$ with $5 \mathrm{~ns}$ beam pulsing by electrostatic sweeper in the low energy section of the accelerator. The latest development for the PWNG is the use of multiple spiral antennas and indirectly water-cooled sapphire windows. The total discharge power of the PWNG unit will be in the order of $5 \mathrm{~kW}$, which is divided to $10 \mathrm{RF}$-windows. Sapphire is used for superior mechanical strength and relatively good heat conductivity. The purpose of the water cooling lines in these brazed window assemblies is to cool the heat generated mainly by the inductive heating of the molybdenum window frames. The PWNG device is shown in Figure 8.

The high voltage switches from BEHLKE ELECTRONIC Gmbh have been assembled in an integrated unit with required to operate the switches. Testing will be done using the previously fabricated prototype system.

Tritium fill will be done at the tritium facility at Livermore National Laboratory. The PWNG unit will be assembled tested and tritium filled during the last quarter 2006.

\section{DISCUSSION}

Plasma and Ion Source Technology Group at Lawrence Berkeley National Laboratory have designed, tested and delivered various types of neutron generator devices for different applications. The neutron yield with high beam power has been extensively studied. New neutron production target and ion source concepts have been developed for high brightness and sealed tube neutron generator applications. Besides the D-D neutron generator development the higher energy $\mathrm{D}-\mathrm{T}$ and/or T-T neutron generations in an axial geometry will be studied in the future for fast through-put explosive detection as well as medical applications.

\section{ACKNOWLEDGEMENTS}

This Work was supported by Tensor Technology Inc., by Adelphi Technology Inc. and by U.S. Department of Energy under contract No. DE-AC02-05CH11231. Author would also like acknowledge the technical and scientific staff of Plasma and Ion Source Technology Group and especially Frederic Gicguel for engineering support, Tak-Pui Lou and Taneli Kalvas for simulations and modeling and Michael King and Hannes Vainionpaa for supplying data for this presentation.

\section{REFERENCES}

[1] J. Reijonen, Proc. Particle Accelerator Conference '05, Knoxville TN, USA, 2005, p. 49

[2] J. Reijonen, F. Gicquel, S. K. Hahto, M. King, T- P Lou and K-N Leung, Appl. Rad. and Isotop, 63, 757 (2005)

[3] Becker, R. and Herrmannsfeldt, W., Rev. Sci. Instrum. 63, 2756 (1992)

[4] "The Stopping and Range of Ions in Solids", by J. F. Ziegler, J. P. Biersack and U. Littmark, Pergamon Press, New York, 1985, http://www.srim.org/

[5] J. T. Cremer, M. A. Piestrup, H. Park, C. K. Gary, R. H. Pantell, C. J. Glinka, and J. G. Barker, Appl. Phys. Lett. 87, 161913 (2005)

[6] H. Vainionpaa, To Be Published

[7] T. G. Miller and R. A. Krauss, Proceedings of the SPIE European Symposium on Optics for Environmental and Public Safety, Munich, 1995 